\title{
Vibrotactile masking: The role of response competition
}

\author{
JAMES C. CRAIG \\ Indiana University, Bloomington, Indiana
}

\begin{abstract}
When two tactile patterns, a target and a nontarget pattern, are presented in close temporal proximity to the same location, the nontarget pattern may interfere with the identification of the target. A series of experiments examined the extent to which the interference in target identification results from masking (interference in the representation of the target at an early stage of processing) or from response competition. A response competition view of pattern perception holds that both the target and nontarget are fully processed to the level of evoking responses. Interference is produced when subjects select the nontarget rather than the target. This view was tested with a paradigm developed in studies of selective attention. Pairs of tactile patterns were presented to subjects' left index fingerpads. The amount of interference produced by a nontarget that is physically different from a target depends on whether the nontarget is associated with the same response as the target or a different response. The amount of masking also depends on the set of target and nontarget patterns that are used. The results support the conclusion that subjects have available a representation of both the target and the nontarget and that a substantial portion of the interference previously attributed to masking may be due to response competition.
\end{abstract}

A number of studies have examined the interaction that occurs between spatio-temporal patterns presented either to the same site or to separate sites on the skin. Several experimental paradigms can be generated from the situation in which there are two patterns presented sequentially, and there are either one or two sites of stimulation. The two patterns may be sequentially presented to the same site, and the subject required to combine them-a temporal integration task (Craig, 1982a, 1984). The two patterns may be presented to separate sites, and the subject required to combine or compare the two patterns--a divided attention task (Craig, 1985). In two other paradigms, the subject is required to identify one of the two patterns and ignore the other. If the two patterns are presented to the same site, this is typically considered a temporal masking paradigm (Bliss, Crane, Link, \& Townsend, 1966; Craig, 1982b, 1983, 1985; Craig \& Evans, 1987; Evans \& Craig, 1986; Schindler \& Knapp, 1976). If the patterns are presented to separate sites, the task becomes one of selective attention (Evans \& Craig, 1991, 1992; Evans, Craig, \& Rinker, 1992; Rinker \& Craig, 1994).

A recent study compared performance in the latter two paradigms, temporal masking and selective attention (Craig \& Evans, 1995). It has generally been thought that rather different processes are involved in temporal masking than in selective attention. In a temporal masking paradigm, there is only one location to which to attend; and, because of this, selective attention was thought not to play much of a

This research was supported by Grant DC-00095 from the National Institutes of Health. The author thanks Roger P. Rhodes for his assistance in conducting these experiments. Reprint requests should be addressed to J. C. Craig, Department of Psychology, Indiana University, Bloomington, IN 47405 (e-mail: craigj@indiana.edu). role. It was considered possible, however, that masking might play some role in a selective attention paradigm when stimuli are presented to separate sites (Evans \& Craig, 1991). Remote site masking in a pattern identification task was considered a possibility, in part because studies have shown that detection of a target stimulus presented to one site can be interfered with by the presentation of a nontarget stimulus (masker) at another site. In some cases, the two stimuli can be as distant as on opposite hands or arms (Gescheider, Herman, \& Phillips, 1970; Gilson, 1969; Sherrick, 1964; Snyder, 1977).

In studies of selective attention with both visual stimuli (Eriksen \& Hoffman, 1973; Eriksen \& Schultz, 1979) and tactile stimuli (Evans \& Craig, 1992), the possibility of masking between a target pattern and a nontarget pattern presented to separate sites has been addressed directly. If in these studies performance declined due to masking, it might lead to the erroneous conclusion that there was a failure of selective attention. That is, performance might decline because the nontarget masked the target, not because the subjects were attending to the nontarget. In this paper, the term masking will be used to refer to interference in the identification of a target pattern that occurs at an early stage of processing. The interference is of the type that distorts the representation of the target pattern. Such a distortion could be the result of temporal integration of the target pattern with the nontarget pattern (Evans, 1987; Evans \& Craig, 1986), distortion of features of the target (Craig, 1989), deletion of target features (Evans \& Craig, 1986), and so forth. Specifically excluded from this use of the term masking is interference in the identification of a target pattern when representations of both the target and the nontarget are available, and the subject mistakenly responds with the nontarget pattern. 
To examine masking effects in the context of selective attention, investigators have used a 4-to-2 paradigm. This paradigm permits one to measure the extent to which masking may interfere with the ability of subjects to identify a target pattern at one location in the presence of a nontarget at a nearby location. In this paradigm, four stimuli are used. On each trial, one of the four stimuli is presented as the target stimulus, but only two responses are provided. If, for example, the subjects receive either pattern A or B, they are to respond " 1. " If they receive C or $D$, they are to respond " 2 ." On each trial, two stimuli-a target and a nontarget stimulus-are presented. The nontarget is selected from the same set of stimuli as is the target. This pairing of the target and the nontarget creates three different types of trials. The target and nontarget may be physically different and associated with a different response (response-incompatible, or RI, trials). The target and nontarget may be physically different and associated with the same response (response-compatible, or $\mathrm{RC}$, trials). The target and nontarget may be physically identical and associated with the same response (stimuluscompatible, or SC, trials). For both visual (Eriksen \& Hoffman, 1973; Eriksen \& Schultz, 1979) and tactile stimuli presented to different sites (Evans \& Craig, 1992; Evans et al., 1992), the finding has been that little interference is seen on either SC trials or RC trials. There is often considerable interference on RI trials; that is, considerable interference results from presenting a nontarget that is not simply physically different from the target but physically different and associated with a different response from that with which the target is associated (RI trials). These results have been used to argue that masking is not a major factor in these studies of selective attention and that both the target and nontarget are fully processed to the point of evoking competing responses. Subjects err not because the representation of the target pattern has been interfered with but because they select and respond with the nontarget.

In a recent study, the time course of selective attention was examined with nontargets presented both before and after the target. More interference was observed with trailing as opposed to leading nontargets (Craig \& Evans, 1995; Rinker \& Craig, 1994). The overall similarity of the interference function to temporal masking functions prompted additional measurements using the 4-to-2 paradigm and the same stimuli, but with the target and nontarget presented to the same location, a temporal masking paradigm. The major finding from these additional measurements was that nontarget stimuli that were physically different from the target but associated with the same response as was the target ( $\mathrm{RC}$ trials) produced only modest interference; whereas the same nontarget, associated with a response different from that for the target (RI trials), produced considerable interference (Craig \& Evans, 1995). In other words, the results were similar to those obtained in the selective attention task. In fact, performance on SC trials was very similar to performance on $\mathrm{RC}$ trials. This result indicated that much of what had been referred to as "masking" might have been due to response competition and not to a phys- ically different stimulus (masker) altering the representation of the target at an early stage of processing.

The possibility has been raised before that some portion of the interference effect produced by a nontarget (masker) might be due to subjects' responding mistakenly with the nontarget (Bliss, Crane, Mansfield \& Townsend, 1966; Craig, 1976; Evans, 1987). In these studies, the target and nontarget were drawn from the same set of patterns; however, even in studies when the nontarget was not drawn from the same set of patterns as was the target, it is possible that the nontarget was evoking target-like responses. In one study of letter identification, the nontargets (maskers) were randomly constructed, letter-like patterns (Craig, 1982a). In other studies, an "energy masker" was used, which is generated by turning on all the pins in the tactile array (Craig, 1978). With the letter-like patterns, it has been argued that although the nontargets were not explicitly associated with particular responses, they are likely to evoke letter responses. Hence, the interference they produce may be due in part to response competition. Energy maskers produce less interference than pattern maskers, presumably because they are less likely to evoke competitive responses; however, even in the case of energy maskers, it has been suggested that this nontarget generates edges and angles (Craig, 1982b), features that might evoke a pattern response. Although the results from earlier studies showed that subjects might err by responding with the nontarget, the studies did not deal explicitly with the issue of response competition and the extent to which subjects have available both the target and nontarget (masker) at the time at which they respond.

In most studies of temporal masking, a single function is obtained, typically some measure of the accuracy of target identification plotted as a function of the temporal separation between the target and masker. With the 4-to-2 paradigm, three functions are obtained, one from each of the three types of trials. In the Craig and Evans (1995) study, when stimuli were presented to the same site, the RI function, which showed the most interference, did not demonstrate maximum interference when the nontarget trailed the target (backward masking). In one condition, there was slightly (nonsignificant) more forward than backward masking, whereas in a second condition, the reverse was the case. A consistent finding in pattern identification tasks is that there is more backward than forward masking (Craig, 1976, 1982b, 1985). The 4-to-2 paradigm differs in several ways from the usual temporal masking paradigm, and it is possible that some of these differences might lead to different temporal masking functions. Equally likely to have produced the differences, though, is the nature of the stimuli used in the Craig and Evans (1995) study. The stimuli were bar-like patterns that appeared to move across the fingerpad. Identification was based on the direction of motion in which the pattern appeared to be moving. It was speculated that presenting a moving target and nontarget in close temporal proximity to the same fingerpad might generate apparent motion between the target and nontarget, which might, in turn, alter the temporal masking function (Craig \& Evans, 1995). 
The present study was designed, first, to extend the earlier findings-specifically, to determine whether response competition was a major factor in temporal masking with the types of stimuli that have generally been used in previous studies with spatial patterns. The majority of these earlier studies used patterns in which pattern identification was based on discriminating spatial features. If response competition were evident only when the direction of motion was the relevant stimulus dimension, its significance in explaining interference between spatial patterns would be considerably reduced. A second aim of the present study was to examine the nature of the representations of the target and nontarget. To what extent do subjects have both the target and nontarget available as responses?

\section{EXPERIMENT 1}

In Experiment 1, the stimuli were line segments presented in different locations on the tactile array. Although generated on a vibrotactile array, the patterns were stationary in that they did not move across the display. These are the types of patterns that have been used in many of the previous temporal masking studies. The 4-to-2 paradigm was used. The first issue to be addressed was whether these stimuli would produce results similar to those obtained with moving stimuli (Craig \& Evans, 1995). The second issue was the nature of the function relating the amount of interference to the temporal separation between target and nontarget. For RI trials, would the function resemble those typically seen in temporal masking studies - that is, more backward than forward masking?

Most of the interference between a target and a nontarget occurs when the two stimuli are presented within $100-150 \mathrm{msec}$ of one another, and it was upon this temporal interval that we focused. However, in a previous study (Craig \& Evans, 1995), an unexpected pattern of results was observed with the 4-to-2 paradigm at long temporal separations. Specifically, increasing the time by which the nontarget led the target from 100 to $500 \mathrm{msec}$ resulted in performance on SC and RC trials declining. It was speculated that these results might be due either to the use of the 4-to-2 paradigm or to the nature of the stimuli themselves, which were moving bar-like patterns. In Experiment 1, measurements were made with stationary stimuli at both brief and longer temporal separations between the target and nontarget. The longer temporal separations were included, to see whether, with stationary patterns as with moving stimuli, target identification performance declined at longer stimulus onset asynchronies (SOAs).

\section{Method}

Subjects. The subjects were students at Indiana University. All subjects were paid for their participation, and all had participated in a number of related studies. In the first set of measurements, 5 subjects were tested. In the second set of measurements, 8 subjects were tested. Two of the latter subjects had participated in the first set of measurements.

Apparatus. Details of the apparatus may be found in Craig (1980). The apparatus consisted of a tactile display from the Optacon. This 144-element display was interfaced with a computer. The computer controlled the tactile display and the duration and sequencing of the tactile patterns, as well as a visual display that was used to present instructions and feedback to the subjects. The computer was also used to collect the subjects' responses.

Stimuli. The stimuli were horizontal and vertical bar-like patterns. For the first set of measurements, each pattern was created by turning on 18 pins for $26 \mathrm{msec}$. For the second set of measurements, the duration was increased to $52 \mathrm{msec}$, the duration that was used in earlier studies with moving stimuli (Craig \& Evans, 1995). The stimuli were presented at a moderate intensity using a driving voltage of $33 \mathrm{~V}$ applied to the stimulators. The stimulators were driven at 230 pps. The stimuli were generated by turning on the left-most or right-most column of the array (top 18 rows) or the top three rows of the array or Rows 16, 17, and 18. The four stimuli contacted the left, right, top, or bottom of the distal portion of the left index fingerpad. Representations of the patterns are shown in Figure 1.

Procedure. The procedure was very similar to that used by Craig and Evans (1995). Each subject was tested individually. The subject rested his/her left index finger on the tactile display. The subjects were instructed that on each trial they would receive two stimuli. The stimulus that they were to respond to, the target stimulus, would be presented either first or second. A message on the CRT, which remained on during a block of trials, informed the subjects which one of the two stimuli, the first or second, was the target. Trials were

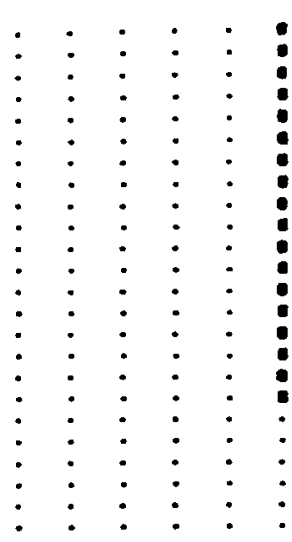

a

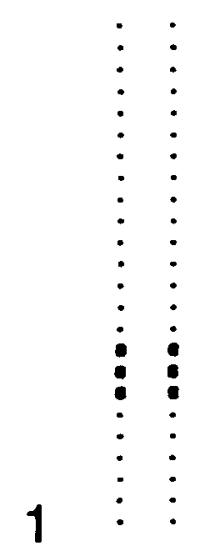

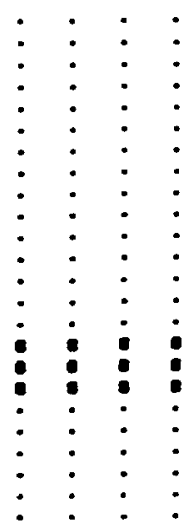

b

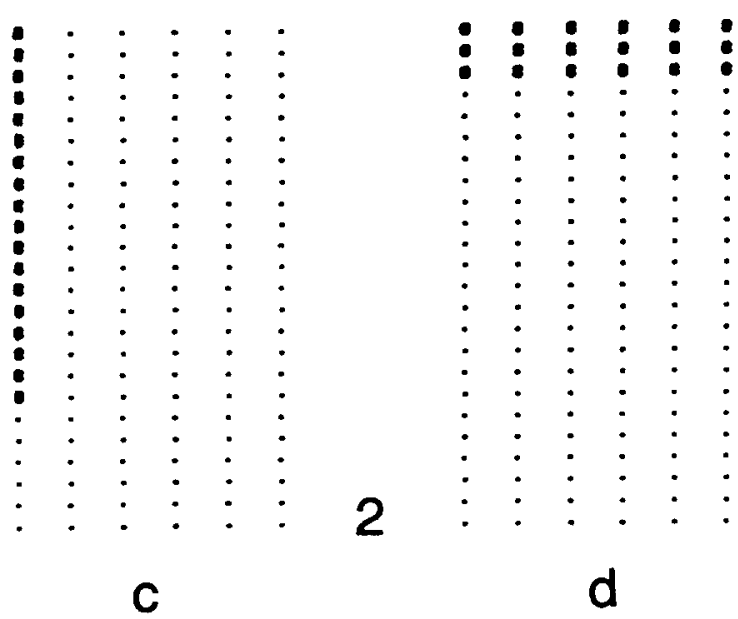

Figure 1. Representations of the stimuli used in the 4-to-2 paradigm in Experiment 1. Stimuli a and $b$ were assigned response " 1 ," and stimuli $\mathrm{c}$ and $\mathrm{d}$ were assigned response " 2 ." 
blocked by SOA, so that subjects knew that for a block of 50 trials they would be attending to either the first or the second pattern and were to disregard the other (nontarget) stimulus.

Subjects were instructed to categorize the four stimuli and respond " 1 " if the target stimulus was either the right vertical or the bottom horizontal pattern and " 2 " if the target was either the left vertical or the top horizontal pattern. The subjects responded by pressing one of two response keys with the index and middle fingers of the right hand. Accuracy was stressed, although the subjects were aware that reaction times were being recorded.

On each trial a target and nontarget were presented. Within a block of trials, target and nontarget patterns were selected randomly. Approximately one fourth of the trials were $\mathrm{SC}$ trials (identical target and nontarget); one fourth of the trials were $\mathrm{RC}$ trials (physically different target and nontarget associated with the same response); the remaining half of the trials were RI trials (physically different target and nontarget associated with different responses).

The subjects initiated each trial by pressing either one of the two response keys. One second later, the stimulus sequence began. Following the presentation of the target and nontarget stimuli, the subjects responded and received feedback via the visual display. A correct response was followed by the word "CORRFCT" appearing on the visual display. An incorrect response was followed by the word "WRONG" appearing on the display. The subjects then initiated the next trial by pressing one of the response buttons. To prevent any auditory cues from the tactile displays influencing subjects' responses, the subjects wore earplugs and headphones through which white noise was presented.

In the first set of measurements, six SOAs between the target and nontarget were tested: $-152,-78,-26,+26,+78$, and $+152 \mathrm{msec}$, where negative SOAs refer to trials in which the nontarget preceded the target, and positive SOAs, the reverse. Each session consisted of seven blocks of 50 trials each. The first block of trials in each session was one in which the target was presented by itself. For the remainder of the session, the blocks of trials testing particular SOAs were selected at random. Subjects were tested for four sessions.

Six SOAs were tested in the second set of measurements: -500 , $-100,-52,+52,+100,+500 \mathrm{msec}$. Subjects were tested for four sessions.

\section{Results and Discussion}

The results from the first set of measurements presented in Figure 2 show the percentage of correct trials as a function of SOA. In the absence of a nontarget, performance was $98 \%$ correct. A two-way, repeated measures analysis of variance (ANOVA) showed a significant effect of SOA $[F(5,35)=12.42, p<.0001]$, a significant effect of trial type $[F(2,14)=45.83, p<.0001]$, and a significant interaction between SOA and trial type $[F(10,70)=7.02$, $p<.0001]$. To see whether the SC and RC trials differed from one another, these data were analyzed excluding the RI trial types. The results of this analysis indicated little or no interference from physically different stimuli associated with the same response as that for the target. Specifically, there was no main effect of trial type $[F(1,4)=$ $5.12, p>.05]$. There was a main effect of SOA $[F(5,20)=$ $3.04, p<.05]$, but no interaction between SOA and trial type $[F(5,20)=0.49, p>.05]$. These results are in agreement with the earlier results obtained with moving stimuli, in that little interference is seen on either SC or RC trials and considerable interference is seen on RI trials. Performance drops to chance levels, $50 \%$ correct, with a backward nontarget. In addition the RI function resembles temporal masking functions obtained in previous studies

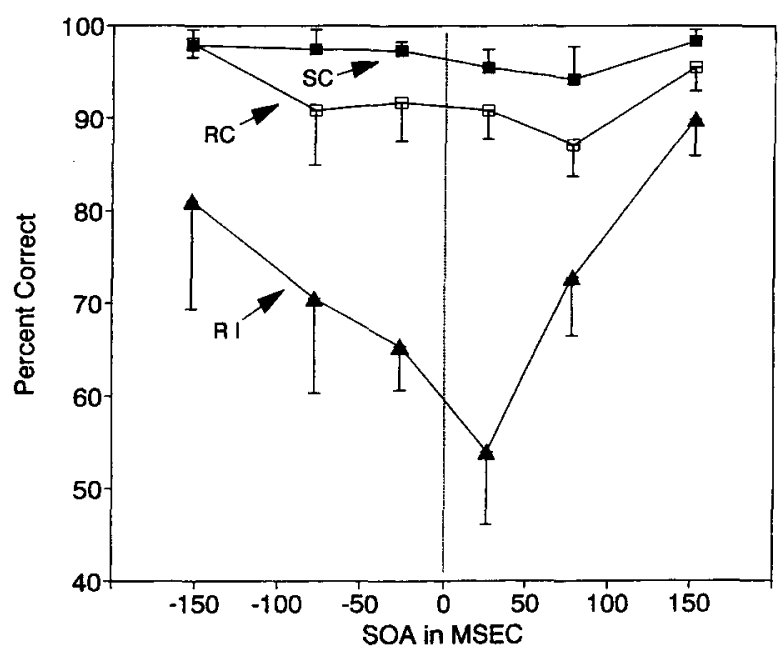

Figure 2. Results from the 4-to-2 paradigm. Percent correct for three trial types-stimulus compatible, response compatible, and response incompatible - as a function of the time between the onset of the nontarget and the onset of the target (SOA). Negative SOAs refer to conditions in which the nontarget precedes the target. Positive SOAs refer to conditions in which the nontarget follows the target. Error bars represent $1 S E M$.

(Craig, 1976; Craig \& Evans, 1987), whereas neither the SC nor the RC function resembles such masking functions.

The second set of measurements is shown in Figure 3. The percentage correct in the absence of a nontarget was $98 \%$. The pattern of results is very similar to that shown in Figure 2. An ANOVA showed a main effect of SOA $[F(5,35)=12.42, p<.0001]$, a main effect of trial type $[F(2,14)=45.83, p<.0001]$, and a significant interaction between SOA and trial type $[F(10,70)=7.02, p<.0001]$. In addition to replicating the effects shown in Figure 2, the results show no evidence of performance declining at longer SOAs, particularly at $-500 \mathrm{msec}$, on either SC or RC trials. These results indicate that previous results from using moving stimuli that did show a decline at $-500 \mathrm{msec}$ for these conditions (Craig \& Evans, 1995) were likely due to the nature of the stimuli that were used and not to the 4-to-2 paradigm.

One explanation for the fact that little interference is seen on RC trials is that for these stimuli a physically different nontarget is not an effective masker; that is, it does not interfere with the target at an early stage of processing. When the nontarget is associated with a response that differs from the target (RI trials), interference increases - a result that is consistent with a response competition explanation. The implication is that representations of both the target and nontarget are available, but the subject selects the nontarget by mistake. Subsequent experiments were designed to test aspects of this view of sequential pattern identification.

\section{EXPERIMENT 2}

In Experiment 2, the numbers of stimuli and responses were increased. One of the limitations of the 4-to-2 para- 


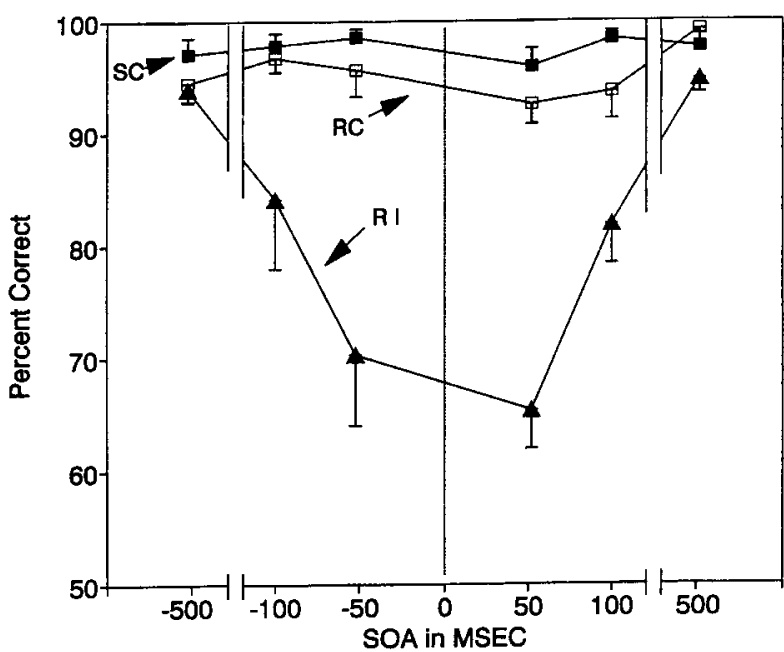

Figure 3. Percent correct for three trial types as a function of stimulus onset asynchrony (SOA). Error bars represent $1 S E M$.

digm is that with only two responses possible, an analysis of which responses subjects choose is typically not definitive. Subjects may err because responses associated with both the target and nontarget are elicited, and subjects have difficulty in deciding on the basis of temporal order which is the target. On the other hand subjects may have available a representation of only one of the two patterns. Even if subjects were aware that the representation that they had available was associated with the nontarget, not the target, their choice would be to respond with the nontarget, which would be correct on some proportion of the trials, or simply guess. With more stimuli and responses available, it is possible to analyze subjects' responses to determine whether, when incorrect, subjects were more likely to choose the nontarget rather than another stimulus.

Reaction time data from an earlier study (Craig \& Evans, 1995) favor the view that subjects have both responses available. Specifically, reaction times, based on correct trials only, were longer on RI than on RC or SC trials. It was argued that reaction times were longer on RI trials because subjects have two representations available signaling different responses. Choosing between these two responses lengthened the reaction times. If subjects had only one response available, reaction times would presumably have been equal on RI, RC, and SC trials.

Increasing the number of stimuli and responses also provides a test of the generality of the results. Most studies of temporal masking have provided subjects with more than two responses. Before concluding that response competition is an important factor in a temporal masking paradigm, it is important to make certain that limiting subjects to two responses does not play a major role in producing the effect.

Experiment 2 consisted of two sets of measurements. In the first set, a 6-to-3 paradigm was used. Subjects were presented with a set of six stimuli and provided with three responses. By analyzing the incorrect responses, one can see whether subjects were selecting the nontarget or were simply guessing. In the second set of measurements, subjects were required to make two responses on every trial. The first response was the subject's best guess as to the target. The second response was the next best guess. Six stimuli were presented, and six responses were provided. An analysis of the first and second responses should indicate whether subjects have representations of both the target and nontarget available when they respond. These measurements specifically address the issue of replacement. If the nontarget is replacing the target in such a way that the subject has only one representation at the time of responding, then, if the first response is correct, the probability of selecting the target on the second response should be at chance levels.

\section{Method}

Subjects. Six subjects were tested in the first set of measurements. Five had participated in Experiment 1. The same 6 subjects were tested in the second set of measurements.

Procedure. The procedure was similar to that used in Experiment 1 The number of stimuli was increased to six. Representations of these stimuli and the way in which subjects were instructed to categorize the stimuli in the first set of measurements are shown in Figure 4.

For the first set of measurements, six SOAs were tested: -152 , $-78,-26,+26,+78$, and +152 msec. As in Experiment 1 , each session consisted of seven blocks of 50 trials, including one block in which the target was presented by itself. Subjects were tested for 10 sessions. The subjects received trial-by-trial feedback indicating whether or not their responses were correct.

In the second set of measurements, the same set of six stimuli was used, but the subjects were trained to give a unique response, from 1 to 6 , for each stimulus. In addition, on every trial, the subjects were required to respond twice. The first response was their best guess as to what target had been presented. The second response was their second best guess. The subjects were prevented from selecting the same pattern for both their first and second responses. The subjects received feedback following the second response, informing them which target had been presented. Two SOAs, one with the nontarget leading the target and one with the nontarget trailing, were selected for testing-specifically, -26 and $+78 \mathrm{msec}$. These two SOAs were selected for testing because the levels of performance at these two SOAs in previous measurements had been comparable. Each testing session consisted of alternating blocks of 50 trials testing the two SOAs. A testing session comprised six blocks. The subjects were tested for eight sessions.

\section{Results and Discussion}

The results from the first set of measurements are shown in Figure 5. The percentage correct for each trial type is plotted as a function of SOA. The percentage correct for target identification in the absence of any masking stimuli was $94 \%$. An ANOVA showed a main effect of SOA $[F(5,25)=7.94, p<.0001]$, a main effect of trial type $[F(2,10)=192.05, p<.001]$, and a significant interaction between SOA and trial type $[F(10,50)=7.42, p<.0001]$.

The results were further analyzed, as they were in Experiment 1 , to see whether SC and RC trials differed from one another. Unlike in the results in Experiment 1, there was a significant effect of trial type $[F(1,5)=24.72, p<$ $.01]$. There was no significant effect of SOA $[F(5,25)=$ $1.55, p>.05]$, nor was there any significant interaction between trial type and SOA $[F(5,25)=1.01, p>.05]$. The fact that performance on RC trials is significantly below 


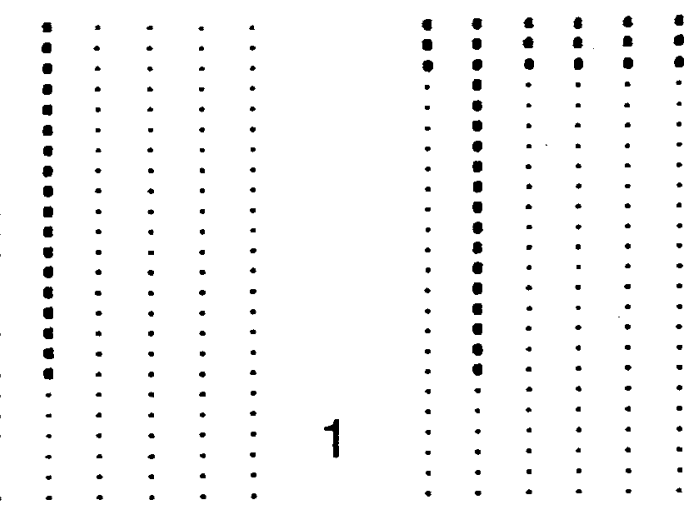

As in Experiment 1, the more significant interference is seen on the RI trials, where performance levels are $15 \%$ $40 \%$ below the performance levels on RC trials. The similarity of the results to those obtained in Experiment 1 indicates that even with an increased number of stimuli and number of responses, response competition continues to appear to play an important part in temporal masking paradigms.

The fact that more interference is evident on RI trials than on RC trials indicates that the subjects have available, and are attempting to respond with, a representation of the nontarget. A further analysis of the responses on RI trials lends support to this contention. The responses on RI trials were analyzed to answer the following question: When responding incorrectly, did subjects respond randomly with one of the two possible responses, or did they select the nontarget more often than the remaining incorrect response? The percentage of RI trials on which subjects selected the nontarget response versus the remaining response (other) is shown in Table 1. The fact that more than twice as many responses were nontarget responses as opposed to other responses offers strong support for the contention that subjects err by responding with the nontarget.

In the second set of measurements, there were six stimuli and six possible responses. These measurements addressed a second issue concerning the information that subjects have available when they respond. Specifically, does the nontarget simply replace the target, or are representations of both available? The measurements addressed this issue by allowing the subjects two responses on each trial. Single pattern performance was relatively good $(90 \%$ correct), but there was a considerable amount of interference. The results of the first-response measurements are shown in Table 2. These results show, as expected, that the most likely response is the correct response; however, correct performance in the presence of nontargets drops to half of what it was in the absence of nontargets. Also, as expected,

Figure 4. Representations of the stimuli used in the 6-to-3 paradigm.

that obtained on SC trials shows that the presence of a physically different nontarget, even one associated with the same response as that for the target, may interfere with target identification. This type of interference should be classified as "masking" and is presumably the result of altering the representation of the target. The fact that in Experiment 1 with four stimuli there was no difference between $\mathrm{SC}$ and RC trials suggests that as either the number or the complexity (or both) of the stimulus set increases, so does the possibility of masking.

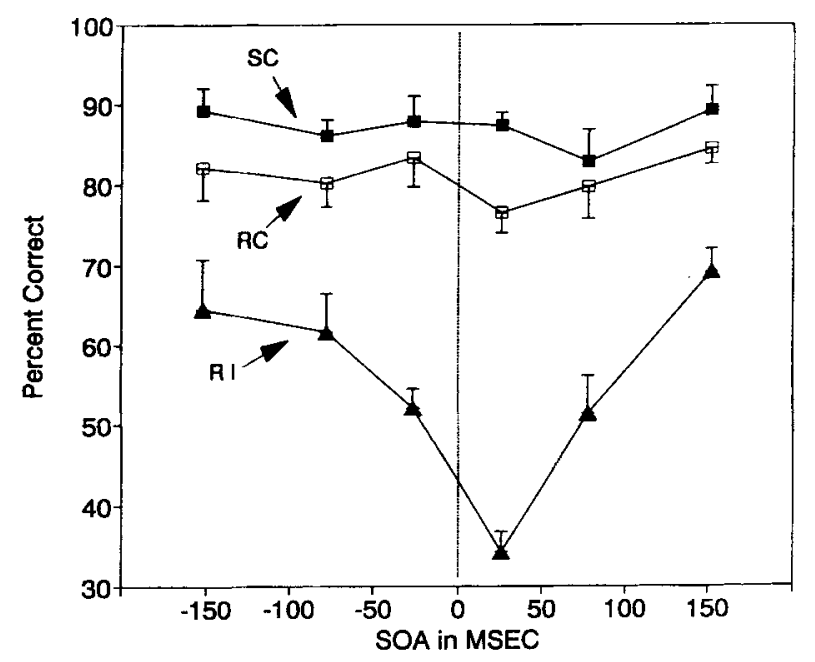

Figure 5. Results from the 6-to-3 paradigm. Percent correct for three trial types as a function of stimulus onset asynchrony (SOA). Error bars represent $1 S E M$. 
Table 1

Percentage of Nontarget Versus Other Responses on Incorrect Trials

\begin{tabular}{lrrrrrr}
\hline & \multicolumn{7}{c}{ SOA } \\
\cline { 2 - 7 } Responses & -152 & -78 & -26 & +26 & +78 & +152 \\
\hline Nontarget & 72 & 71 & 62 & 73 & 74 & 71 \\
Other & 28 & 29 & 38 & 27 & 26 & 29 \\
\hline
\end{tabular}

Note-SOA, stimulus onset asynchrony (in milliseconds)

the nontarget responses are above chance and the responses involving the remaining four patterns-the ones not presented on a particular trial (other) - are below chance. When incorrect, a large proportion of the responses are nontarget responses. For example, in the -26 -msec condition, $40 \%$ of the incorrect responses are nontarget responses, whereas chance would be 1 in 5 or $20 \%$. A chisquare test was performed on the incorrect responses. It showed that the number of nontarget responses at both SOAs exceeded that expected by chance $(p<.001)$.

The data from the second-response results are shown in Table 3. To analyze these data, the trials were divided into three types, depending on the nature of the first responsethat is, on whether the subject's first response was that for the target, the nontarget, or one of the other four responses. For example, at $-26 \mathrm{msec} \mathrm{SOA}$, when the first response was associated with the target, $29.9 \%$ of the second responses were associated with the nontarget and $70.1 \%$ of the second responses with one of the other patterns. An examination of Table 3 shows that in all cases the subjects were above chance in selecting either the target or the nontarget in preference to making one of the other responses. A chi-square test showed that the number of responses for the patterns presented (either target or nontarget) exceeded that expected by chance $(p<.001)$. This was the case whether the first response was associated with the target or the nontarget, or was one of the other responses. When their first response is incorrect, subjects do not respond randomly. Moreover, it does not appear as though the nontarget replaces the target, but rather subjects have available both the target and nontarget when they respond.

\section{EXPERIMENT 3}

A larger and more complex set of patterns was used in Experiment 3: 10 letters of the alphabet. Increasing the number of patterns from four to six (Experiment 1 to Experiment 2) resulted in a significant difference between the $\mathrm{SC}$ and $\mathrm{RC}$ functions, which was presumably a result of an increase in masking. A further increase in size of the pattern set to 10 might enhance the difference between SC and RC functions, if the number of patterns was a major factor in determining the amount of masking. Also, in much of the previous work on spatial pattern perception and on masking, letters have been used as the target patterns (Loomis \& Lederman, 1986). Letters have been used because subjects already know the response code and because letters are varied and complex enough so that subjects have to base their identification, at least in part, on spatial codes (Vega-Bermudez, Johnson, \& Hsaio, 1991). Previous studies have suggested that masking may involve alteration of the spatial features of the target pattern (Craig, 1989; Evans, 1987; Evans \& Craig, 1986). The larger set of patterns might provide a greater opportunity for masking and also test the generality of the findings from Experiments 1 and 2.

\section{Method}

Subjects. Eight subjects were tested. Six had not participated in any of the previous experiments.

Procedure. The stimuli were the first 10 letters of the alphabet, excluding I, J, K, and L, which are relatively easy to identify (Craig, 1979). The 10 letters were mapped onto five responses: $A$ or $B$ was 1 ; C or D, 2; E or F, 3; G or H, 4; and M or N, 5. The subjects were provided with a card showing the letters and response categories to use in responding. The subjects received feedback as to whether their responses were correct or incorrect.

As before, pairs of patterns were selected at random, one pattern as target and the other as nontarget. Random selection was necessary so that the nontarget conveyed no information about the target. This random selection resulted in an average of $10 \%$ of the trials being $\mathrm{SC}$ trials, $10 \% \mathrm{RC}$, and $80 \% \mathrm{RI}$. The procedure was similar to that used in Experiment 1. Four SOAs were tested: $-152,-52,+52$, and $+152 \mathrm{msec}$. The subjects were tested for five blocks per session and 21 sessions.

\section{Results and Discussion}

Single letter performance was $72 \%$ correct. Performance in the presence of nontargets is presented in Figure 6. The pattern of results is very similar to that seen in Experiment 1 , with SC and RC performance similar to one another and RI performance considerably poorer. The ANOVA showed a significant effect of SOA $[F(3,21)=7.26, p<.01]$, a significant effect of trial type $[F(2,14)=121.75, p<.0001]$, and a significant interaction $[F(6,42)=5.83, p<.001]$.

Table 2

First Response in Two-Response Paradigm

\begin{tabular}{ccccccccc}
\hline & \multicolumn{2}{c}{ Target } & & \multicolumn{2}{c}{ Nontarget } & & \multicolumn{2}{c}{ Other } \\
\cline { 2 - 3 } \cline { 7 - 8 } SOA & $\%$ & Chance $\%$ & & $\%$ & Chance $\%$ & & Chance $\%$ \\
\hline$-26 \mathrm{msec}$ & 45.3 & 17 & & 21.7 & 17 & & 33.0 & 67 \\
$+78 \mathrm{msec}$ & 43.9 & 17 & & 29.6 & 17 & & 26.5 & 67 \\
\hline
\end{tabular}

Note-SOA, stimulus onset asynchrony; $\%$, percentages by condition: Chance $\%$, percentages expected by chance.

Table 3

Second Responses in Two-Response Paradigm

\begin{tabular}{|c|c|c|c|c|c|c|}
\hline \multirow{3}{*}{$\begin{array}{c}\text { First } \\
\text { Response }\end{array}$} & \multicolumn{6}{|c|}{ Second Response } \\
\hline & \multicolumn{2}{|c|}{ Target } & \multicolumn{2}{|c|}{ Nontarget } & \multicolumn{2}{|r|}{ Other } \\
\hline & $\%$ & Chance \% & $\%$ & Chance $\%$ & $\%$ & Chance \% \\
\hline \multicolumn{7}{|c|}{$-26-\mathrm{msec} \mathrm{SOA}$} \\
\hline Target & & & 29.9 & 20 & 70.1 & 80 \\
\hline Nontarget & 41.6 & 20 & & & 58.4 & 80 \\
\hline Other & 42.8 & 20 & 23.8 & 20 & 33.2 & 60 \\
\hline \multicolumn{7}{|c|}{$+78-\mathrm{msec} \mathrm{SOA}$} \\
\hline Target & & & 36.2 & 20 & 63.8 & 80 \\
\hline Nontarget & 37.5 & 20 & & & 62.5 & 80 \\
\hline Other & 32.6 & 20 & 36.9 & 20 & 30.5 & 60 \\
\hline
\end{tabular}

Note-- \%, percentages by condition; Chance \%, percentages expected by chance; SOA, stimulus onset asynchrony. 


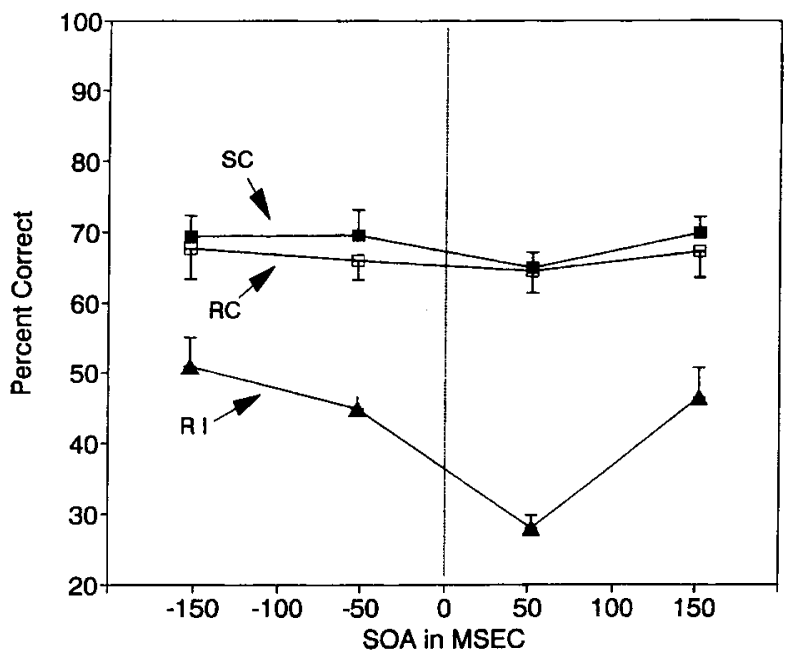

Figure 6. Results from Experiment 3 with letter patterns. Percent correct for three trial types as a function of stimulus onset asynchrony (SOA). Error bars represent $1 S E M$.

The $\mathrm{SC}$ and $\mathrm{RC}$ data were subjected to a separate ANOVA which showed no effect of SOA $[F(3,21)=1.48, p>.05]$, no effect of trial type $[F(1,7)=4.03, p>.05]$, and no significant interaction between SOA and trial type $[F(3,21)=$ $0.21, p>.05]$. Increasing the set size and the complexity of the patterns does not increase the difference between $\mathrm{SC}$ and $\mathrm{RC}$ trials. It appears that there is little masking and that the major source of interference with these patterns is response competition. These results suggest that in reading with the Optacon performance is limited less by masking and more by response competition. At high reading rates, processing the order of the letters may be more of a problem than the representation of the individual letters.

\section{EXPERIMENT 4}

The results from Experiments 1, 2, and 3 suggest that a considerable portion of the interference between successive, spatial patterns is due to response competition, not to masking. The stimuli tested ranged from very simple patterns (Experiment 1) to fairly complex spatial patterns (letters in Experiment 3). With simple, highly identifiable patterns it is perhaps not surprising that little masking might be seen. It is more surprising that no masking was seen with letters. Single-letter identification was well below $100 \%$ correct. Letters, however, may not provide the most sensitive indicator of masking. Letters are highly redundant. Even if certain parts of a letter are obscured, there may be sufficient information to correctly identify the pattern. In several earlier studies, a set of patterns was used that required that each feature of the pattern be perceived for the pattern to be correctly identified (Evans, 1987; Evans \& Craig, 1986). Considerable interference was seen with these patterns. In Experiment 4, four of these patterns, consisting of two line segments each, were used. The patterns were constructed in such a way that both line seg- ments and their spatial positions needed to be perceived correctly for one to identify the pattern.

\section{Method}

Subjects. Five subjects were tested. All had participated in previous experiments.

Procedure. The procedure was similar to that used in Experiment 1 . The major difference was the change in the set of patterns. Representations of the patterns are shown in Figure 7. The patterns were constructed so that the position of both line segments had to be correctly perceived in order to produce a correct response. The two patterns in each category were grouped so that they shared neither of the two features of the other patterns. If the subject perceived only one of the two line segments in a pattern, the pattern would likely resemble one of the two patterns from the other category-an incorrect response. The pattern set was designed to reveal masking effects should they exist. Six SOAs, ranging from -152 to $+152 \mathrm{msec}$, were tested. The subjects were tested for four sessions.

\section{Results and Discussion}

The results are shown in Figure 8. Single pattern performance was $88 \%$ correct. Performance in the presence

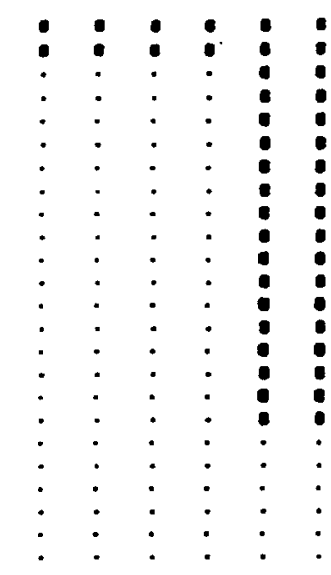

a

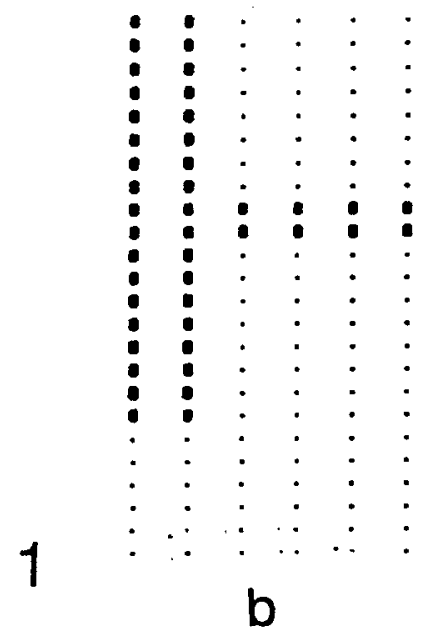

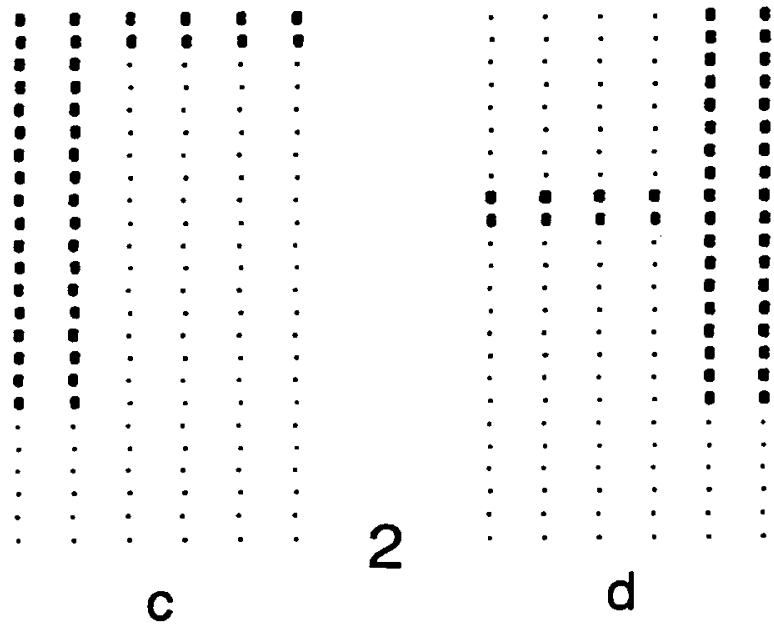

Figure 7. Representations of the stimuli used in Experiment 4. 
of a nontarget differed from that found in Experiments 1, 2 , and 3 . With this pattern set, RC performance is considerably below SC performance and much more similar to RI performance. An ANOVA performed on the overall results showed a significant effect of SOA $[F(5,20)=4.17$, $p<.01]$, a significant effect of trial type $[F(2,8)=59.79$ $p<.0001]$, and a significant interaction between SOA and trial type $[F(10,40)=2.93, p<.01]$.

The most important difference between the earlier functions and the ones shown in Figure 8 is the reduced performance seen on RC trials. In Figure 2, the average difference between $\mathrm{SC}$ and $\mathrm{RC}$ trials was $5 \%$. The comparable figure for the more complex patterns in Experiment 4 is $22 \%$. It is clear that, with this pattern set, physically different patterns produce much more interference than that seen with the other pattern sets. An ANOVA on the data from the RC and RI trials supports the observation that RC and RI performance levels are similar. There was no significant effect of trial type $[F(1,4)=4.21, p>.05]$. There was a significant effect of SOA $[F(5,20)=5.67, p<.01]$. There was no significant interaction between SOA and trial type $[F(5,20)=1.88, p>.05]$.

With this pattern set, it appears that masking plays a major role in producing interference. Performance levels on RI trials are close to chance levels, $50 \%$ correct. It is possible that a larger set of patterns would produce lower levels of chance performance and might allow one to see differences between RC and RI performance. If masking is the sole reason for lowering performance on $\mathrm{RC}$ trials, then, logically, performance can only drop as low as chance levels. If the representation of the target is totally obscured, subjects could only guess among available alternatives. On the other hand, if response competition is playing some part, performance could go lower than chance as the subject attempts to respond with the nontarget.

As noted above, masking might be seen with the patterns used in Experiment 4 because both of the line seg-

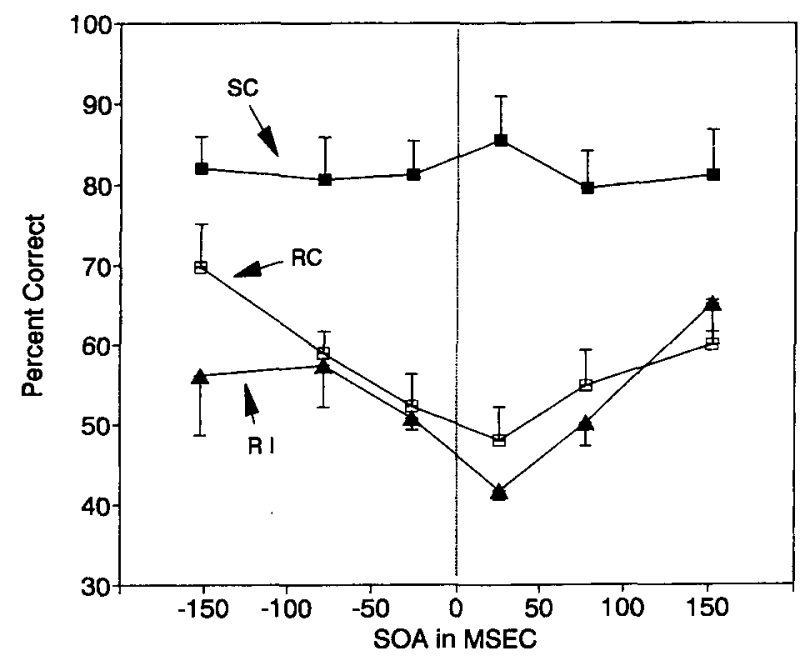

Figure 8. Results with more complex stimuli in 4-to-2 paradigm. Percent correct for three trial types as a function of stimulus onset asynchrony (SOA). Error bars represent 1 SEM. ments making up the patterns need to be perceived in order for one to correctly categorize the patterns, and failure to perceive one of the line segments would likely result in the target pattern's resembling a pattern from the incorrect category. Temporal integration could also be playing a role with these patterns. If the target and nontarget are integrated to form a single pattern, the target might be difficult to identify (Evans, 1987; Evans \& Craig, 1986). On RC trials, both patterns a and b would be presented (Figure 7) or $\mathrm{c}$ and $\mathrm{d}$. The pattern resulting from the temporal integration of $a$ and $b$ is identical to the pattern produced by the temporal integration of $c$ and $d$. The fact that identical patterns would be produced on RC trials with stimuli from Category 1 or Category 2 might result in a considerable number of errors, as indeed there are. Temporal integration could also be playing a role in the results from Experiments 1, 2, and 3. If the integrated patterns, consisting of the target and nontarget, were reasonably simple, such as those that would be generated in Experiments 1 and 2, subjects might learn to identify them. This strategy would be particularly helpful on RC trials on which the temporal order of the target and nontarget was irrelevant, since both signal the same response.

The more complex patterns used in Experiment 3, ten letters, provide a more stringent test of a temporal integration model. The model would have to maintain that pairs of different letters within a category, such as A and B, when integrated into a single pattern, are as readily identified as pairs of identical letters. The model needs to maintain this, because performance on RC trials is nearly identical to performance on SC trials (Figure 6). It seems unlikely that complex patterns formed by combining $\mathrm{A}$ and $\mathrm{B}$ or $\mathrm{C}$ and $\mathrm{D}$ or $\mathrm{G}$ and $\mathrm{H}$ would be as easily identified as pairs of identical letters. Perhaps only certain letter pairs on RC trials, such as EF and MN, combine to form integrated patterns that are simple enough to be as readily identified as the integrated pairs of identical letters. The results from the RC and SC trials in Experiment 3 were analyzed to determine whether there were large differences between the letter pairs EF and $\mathrm{MN}$ and the remaining three pairs of letters. There was a slight decline in performance on $\mathrm{RC}$ as compared with $\mathrm{SC}$ trials for both groups of letters, but this decline was almost the identical for the two groups of letters: $2.6 \%$ for $\mathrm{EF}$ and $\mathrm{MN}$, and $3.1 \%$ for $A B, C D$, and $G H$. These results suggest that temporal integration is not playing a major role in the letter identification task. Because temporal integration appears to be a pervasive phenomenon with tactile spatial patterns, it is possible that it is playing a role with some of the pattern sets in the present study. Additional measurements with other pattern sets will be necessary in order to determine how significant that role is.

\section{GENERAL DISCUSSION}

The present results indicate that when a sequence of patterns is presented to the same location a major difficulty in identifying a target pattern is response competition. Representations of the patterns - both target and nontar- 
get--are available, but subjects err by selecting the wrong response. Previous results have also shown that subjects may respond with a nontarget (masker) (Evans, 1987). What the present results show is that such "masker responses" may be very likely under certain circumstances.

The amount of interference that results from response competition is reflected in the difference in performance levels between the RC and RI functions. The decrement in performance on RI trials is due, presumably, to subjects' making nontarget responses. This decrement appears to be greatest when the target and nontarget are separated by $150 \mathrm{msec}$ or less and greater when the nontarget trails by $50 \mathrm{msec}$ rather than leads by $50 \mathrm{msec}$. The temporal course of the response competition effect is very similar to that seen in temporal masking functions in general. To the extent that many temporal masking functions have a response competition component, such a similarity is to be expected. For example, when the target and nontarget (masker) are drawn from the same set of patterns, much of the interference may be due to response competition, and the function relating performance to the temporal separation of target and nontarget might well resemble the function obtained from measures of response competition. However, even when targets and nontargets are drawn from different sets of patterns or an energy masker is used, thus reducing the amount of response competition (Craig, $1982 \mathrm{~b}$ ), the shape of the interference function remains about the same. The general conclusion is that the time course of masking and the time course of response competition are similar.

In the absence of the results of Experiment 4, one might be tempted to assert that nontarget stimuli do not mask target stimuli-that is, that they do not interfere with target stimuli at an early stage of processing. The results of Experiment 4 demonstrate that such masking does take place and can lead to a significant reduction in performance. The stimulus conditions in Experiment 4 are identical to the conditions in Experiments 1, 2, and 3, with the exception of the set of patterns that was used. Hence, it may be that the nontarget is altering the representation of the target in the earlier experiments even in conditions in which performance levels are unaffected. What this suggests is that more subtle measures of performance might reveal effects of nontarget stimuli, even when identification accuracy is unaffected. One might find changes in the kinds of confusions that subjects make or changes in reaction times. It might be possible to increase the number of patterns within a category to three or more. With an increase in the number of nontargets signaling the same response, one could ask subjects to both categorize and identify the target stimulus. Certain combinations of targets and nontargets on RC trials might lead to good categorization but poor identification performance. Such a result would suggest that the nontarget is altering the representation of the target but not enough to cause subjects to miscategorize it.

The present results have implications for neurophysiological studies that have examined how tactile spatial patterns are represented in the nervous system. These studies have produced reconstructed neural images based on both peripheral recordings and central recordings in SI (Phillips, Johansson, \& Johnson, 1990; Phillips, Johnson, \& Hsiao, 1988). At present, researchers have examined interactions only between relatively simple patterns; however, as both stimulus control and recording techniques become more sophisticated, it should be possible to present pairs of spatial patterns in rapid succession to the same area of skin. Reconstructed neural images may provide information about how the spatial image of a target pattern is altered and at what level in the nervous system such an alteration takes place.

Previous studies of temporal masking have stressed how the representation of a target pattern is changed as a result of close temporal proximity to a nontarget (masking) pattern. The discussion in earlier studies has focused on the lack of a clear representation of the target or an altered representation of the target. If the target representation were altered at an early stage of processing, performance would be poor because the target does not evoke a correct response. To improve performance one would concentrate on ways to improve the representation of the target. On the other hand, if, as the present results suggest, the target is capable of evoking a correct response and performance is poor because the wrong response is selected, the focus shifts to a consideration of ways to improve response selection. One way to improve response selection would be to provide subjects with information about the sequence of patterns that is to be presented. It may be that modest amounts of information in the form of redundancy, schemas, or knowledge about an object would provide major gains in pattern identification.

\section{REFERENCES}

Bliss, J. C., Crane, H. D., Link, S. W., \& Townsend, J. T. (1966). Tactile perception of sequentially presented spatial patterns. Perception \& Psychophysics, 1, 125-130.

Bliss, J. C., Crane, H. D., Mansfield, P. K., \& Townsend, J. T. (1966). Information available in brief tactile presentations. Perception \& Psychophysics, 1, 273-283

Craig, J. C. (1976). Vibrotactile letter recognition: The effects of a masking stimulus. Perception \& Psychophysics, 20, 317-326.

CraIG, J. C. (1978). Vibrotactile pattern recognition and masking. In G. Gordon (Ed.), Active touch: The mechanism of recognition of objects by manipulation (pp. 229-242). Oxford, U.K.: Pergamon.

Craig, J. C. (1979). A confusion matrix for tactually presented letters. Perception \& Psychophysics, 26, 409-411.

CraIG, J. C. (1980). Modes of vibrotactile pattern perception. Journal of Experimental Psychology: Human Perception \& Performance, 6, 151166.

Craig, J. C. (1982a). Temporal integration of vibrotactile patterns. Perception \& Psychophvsics, 32, 219-229.

Craig, J. C. (1982b). Vibrotactile masking: A comparison of energy and pattern maskers. Perception \& Psychophysics, 31, 523-529.

Craig, J. C. (1983). The role of onset in perception of sequentially presented vibrotactile patterns. Perception \& Psychophysics, 34, 421432.

Craig, J. C. (1984). Vibratory temporal integration as a function of pattern discriminability. Perception \& Psychophysics, 35, 579-582.

Craig, J. C. (1985). Tactile pattern perception and its perturbations. Journal of the Acoustical Society of America, 77, 238-246.

Craig, J. C. (1989). Interference in localizing tactile stimuli. Perception \& Psychophysics, 45, 343-355.

Craig, J. C., \& Evans, P. M. (1987). Vibrotactile masking and the persistence of tactual features. Perception \& Psychophysics, 42, 309-317. 
Craig, J. C., \& Evans, P. M. (1995). Tactile selective attention and temporal masking. Perception \& Psychophysics, 57, 511-518.

ERIKSEN, C. W., \& HoFFMAN, J. E. (1973). The extent of processing of noise elements during selective encoding from visual displays. Perception \& Psychophysics, 14, 155-160.

ERIKSEN, C. W., \& SCHUltz, D. W. (1979). Information processing in visual search: A continuous flow conception and experimental results. Perception \& Psychophysics, 25, 249-263.

Evans, P. M. (1987). Vibrotactile masking: Temporal integration, persistence, and strengths of representations. Perception \& Psychophysics, 42, 515-525.

Evans, P. M., \& Craig, J. C. (1986). Temporal integration and vibrotactile backward masking. Journal of Experimental Psychology: Human Perception \& Performance, 12, 160-168.

Evans, P. M., \& Craig, J. C. (1991). Tactile attention and the perception of moving tactile stimuli. Perception \& Psychophysics, 49, 355-364.

Evans, P. M., \& Craig, J. C. (1992). Response competition: A major source of interference in a tactile identification task. Perception \& Psychophysics, 51, 199-206.

Evans, P. M., Craig, J. C., \& Rinker, M. A. (1992). Perceptual processing of adjacent and nonadjacent tactile nontargets. Perception \& Psychophysics, 52, 571-581.

Gescheider, G. A., Herman, D. D., \& Phillips, J. N. (1970). Criterion shifts in the measurement of tactile masking. Perception \& Psychophysics, 8, 433-436.

Gilson, R. D. (1969). Vibrotactile masking: Some spatial and temporal aspects. Perception \& Psychophysics, 5, 176-180.
Loomis, J. M., \& Lederman, S. J. (1986). Tactual perception. In K. R. Boff, L. Kaufman, \& J. P. Thomas (Eds.), Handbook of perception and human performance (pp. 31-4 to 31-41). New York: Wiley.

Phillips, J, R., Johansson, R. S., \& Johnson, K. O. (1990). Representation of Braille characters in human nerve fibers. Experimental Brain Research, 81, 589-592.

Phillips, J. R., Johnson, K. O., \& Hsiao, S. S. (1988). Spatial pattern representation and transformation in monkey somatosensory cortex. Proceedings of National Academy of Sciences, 85, 1317-1321.

Rinker, M. A., \& Craig, J. C. (1994). The effect of spatial orientation on the perception of moving tactile stimuli. Perception \& Psychophysics, 56, 356-362.

SCHINDLER, U., \& KNAPP, A. (1976). Ursachen der gegenseitegen Verdeckung von taktil dargebotenen Buchstaben: Unterbrechung, Summation oder Verzogerung? [The causes of mutual masking of tactile letters: Interruption, summation, or delay?] Psychological Research, 46, 721-739.

SHerrick, C. E. (1964). Effects of double simultaneous stimulation of the skin. American Journal of Psychology, 77, 42-53.

SNYDER, R. E. (1977). Vibrotactile masking: A comparison of psychophysical procedures. Perception \& Psychophysics, 22, 471-475.

Vega-Bermudez, F., Johnson, K. O., \& Hsaio, S. S. (1991). Human tactile pattern recognition: Active versus passive touch, velocity effects, and patterns of confusion. Journal of Neurophysiology, 65, 531-546.

(Manuscript received December 28, 1994; revision accepted for publication April 26, 1995.) 